\title{
Dehalococcoides mccartyi strain GEO12 has a natural tolerance to chloroform inhibition
}

\author{
Chang Ding ${ }^{\dagger+}$, Matthew J. Rogers ${ }^{+}$, and Jianzhong He * \\ $¥$ These two authors contributed equally to this work.
}

Department of Civil and Environmental Engineering, National University of Singapore, Singapore 117576

$\dagger$ Present address: Department of Isotope Biogeochemistry, Helmholtz-Centre for Environmental Research - UFZ, Leipzig, Germany, 04318

Supporting Figures .52

Supplementary Figure S1. 16S-DGGE profile suggests culture purity of Dehalococcoides mccartyi strain GEO12.

Supplementary Figure S2. Gene clusters of $v c r A(A)$ and tceA (B) in the draft genome of strain GEO12.

Supplementary Figure S3. Dechlorination of various chlorinated compounds by Dehalococcoides mccartyi strain GEO12 and cell growth curves.

Supplementary Figure S4. Transcription of $v c r A$ and tceA genes during dechlorination of transdichloroethene (trans-DCE) by Dehalococcoides mccartyi strain GEO12.

Supplementary Figure S5. Transcription of vcrA and tceA genes during dechlorination of 1,2dichloroethane (1,2-DCA) by Dehalococcoides mccartyi strain GEO12.

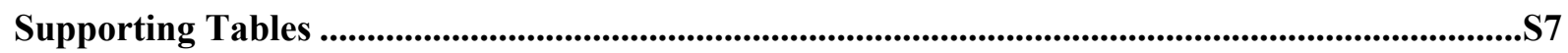

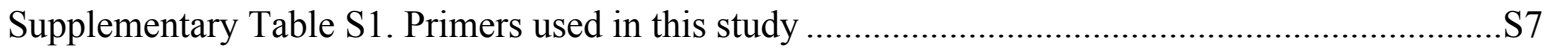

Supplementary Table S2. Bootstrap hypothesis testing of gene expression ...................................S8

Supplementary Table S3. Average nucleotide identity calculated using MUMMer (ANIm) and DNADNA hybridization (DDH) values of Dehalococcoides genomes compared to the genome of Dehalococcoides mccartyi strain GEO12. . ...S9

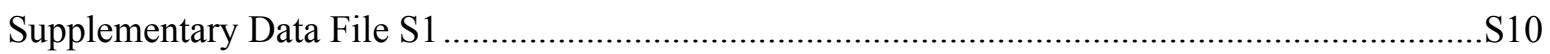

References . $\mathrm{S10}$

Total pages: 10; total figures: 5 ; total tables: 3 ; total data files: 1 


\section{Supporting Figures}

Supplementary Figure S1. 16S-DGGE profile suggests culture purity of Dehalococcoides mccartyi strain GEO12.

Lanes 1, 4, 7, 8, 9 are from gDNA of known pure cultures. Note the minor bands that are always associated with the major bands. Lane 1: Sulfurospirillum multivorans. Lane 2: Dehalococcoides sp. strain DG-dominated mixed culture. Lane 3: a Desulfovibrio-dominated mixed culture. Lane 4: Acetobacterium malicum strain DSM4132. Lane 5: a PBDE-degrading mixed culture. Lane 6: a Desulfitobacterium-dominant mixed culture. Lane 7: Dehalococcoides mccartyi strain ANAS2. Lane 8: Dehalococcoides mccartyi strain MB. Lane 9: Dehalococcoides mccartyi strain 195. Lane 10: Dehalococcoides mccartyi strain GEO12.

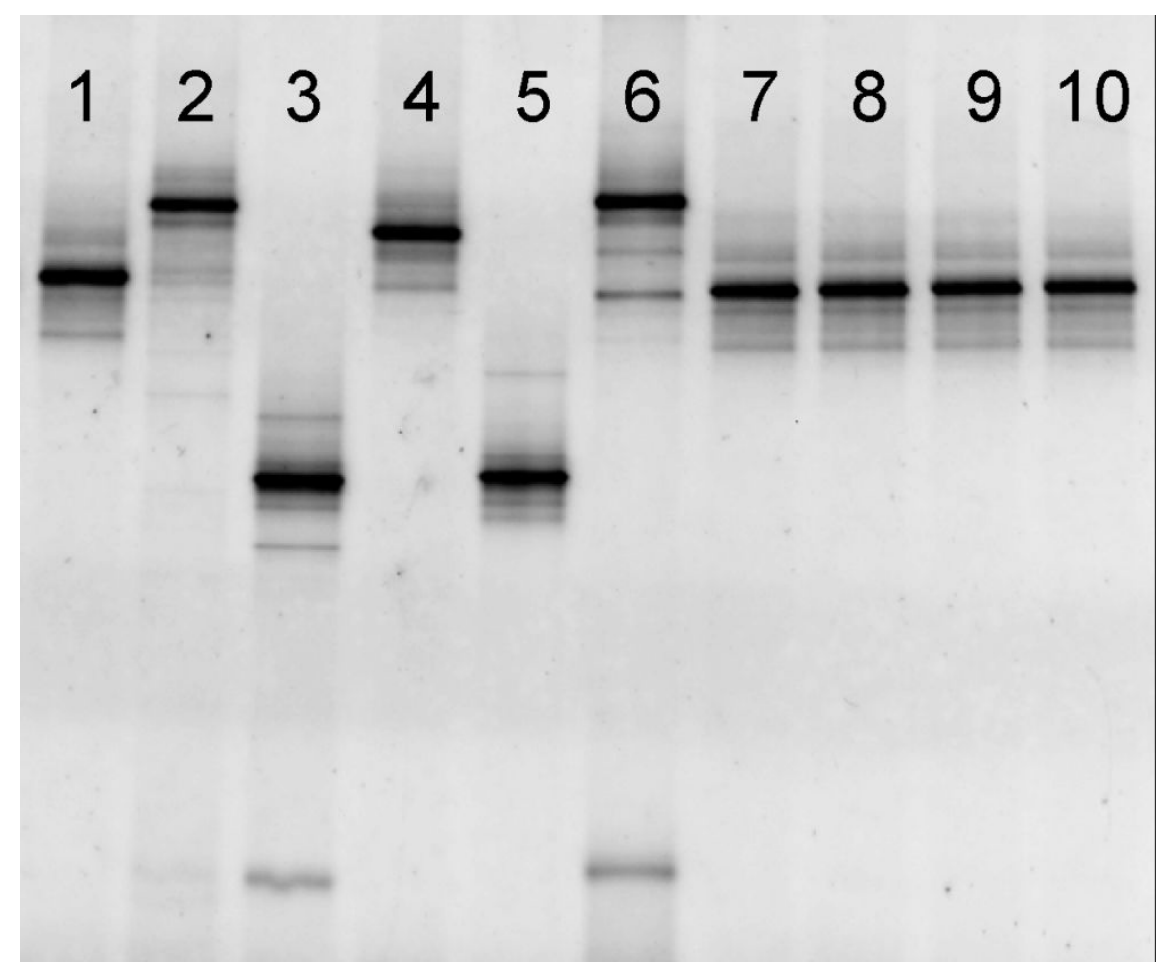


Supplementary Figure S2. Gene clusters of $v c r A$ (A) and tceA (B) in the draft genome of strain GEO12. Unlabeled genes are those predicted to produce hypothetical proteins.

A

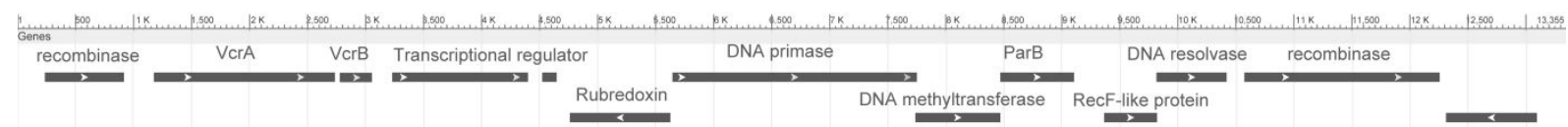

B

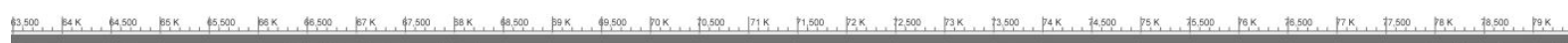
DNA helicase 
Supplementary Figure S3. Dechlorination of various chlorinated compounds by Dehalococcoides mccartyi strain GEO12 and cell growth curves.

Cultivation was done in $160-\mathrm{mL}$ serum bottles containing $100 \mathrm{~mL}$ minimal salts medium. (A) TCE; (B) 1,2-DCA; (C) trans-DCE; (D) cis-DCE; (E) 1,1-DCE. Based on the genomic information of strain GEO12, 16S rRNA gene copies were converted to cell numbers at one copy per cell.
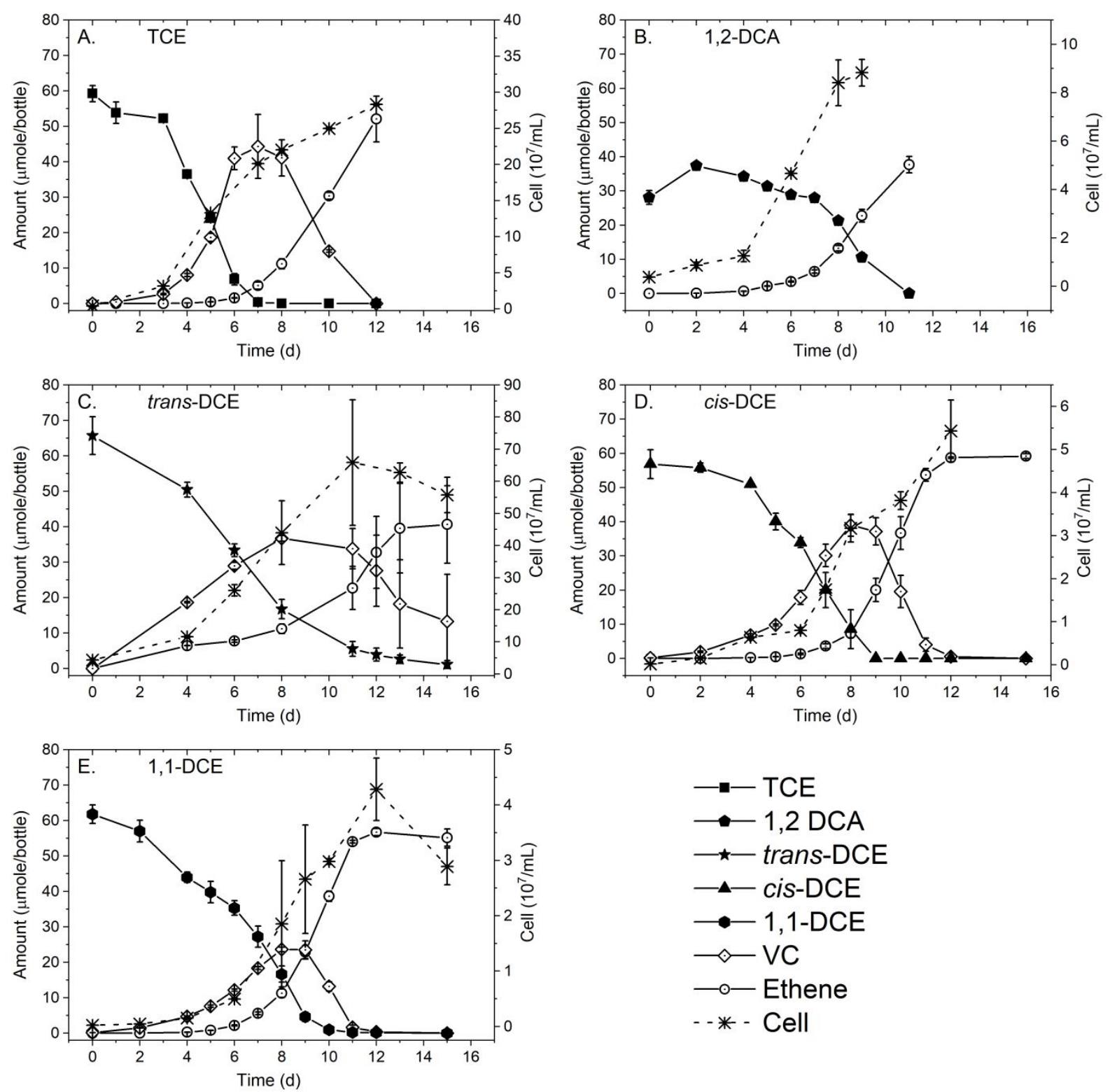
Supplementary Figure S4. Transcription of $v c r A$ and tceA genes during dechlorination of trans-DCE by Dehalococcoides mccartyi strain GEO12.

Cultivation was done in $160-\mathrm{mL}$ serum bottles containing $100 \mathrm{~mL}$ minimal salts medium. Upper panel: transcription of $v c r A$ and $t c e A$ genes during dechlorination of trans-DCE. Lower panel: dechlorination of trans-DCE and cell growth.
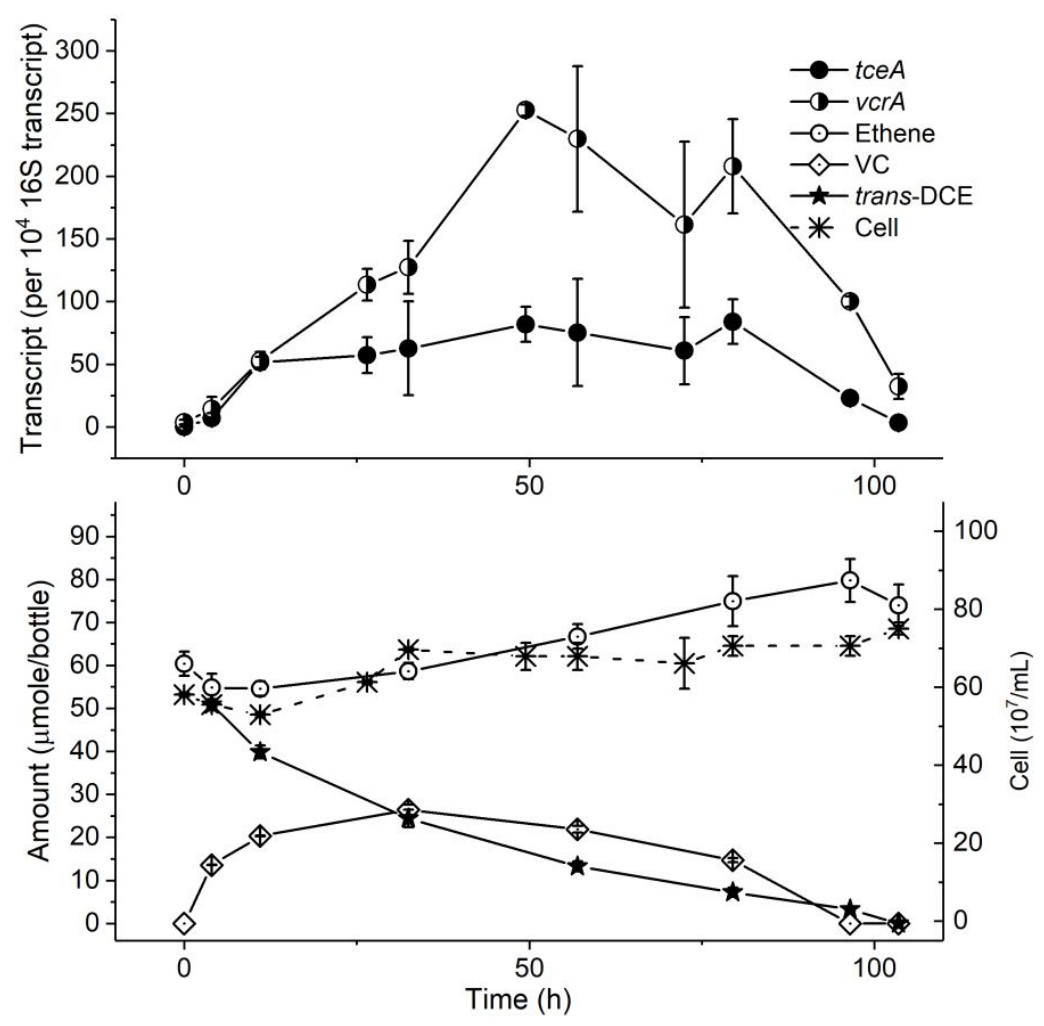
Supplementary Figure S5. Transcription of $v c r A$ and tceA genes during dechlorination of 1,2-DCA by Dehalococcoides mccartyi strain GEO12.

Cultivation was done in $160-\mathrm{mL}$ serum bottles containing $100 \mathrm{~mL}$ minimal salts medium. Upper panel: transcription of $v c r A$ and tce $A$ genes during dechlorination of 1,2-DCA. Lower panel: dechlorination of 1,2-DCA and cell growth.

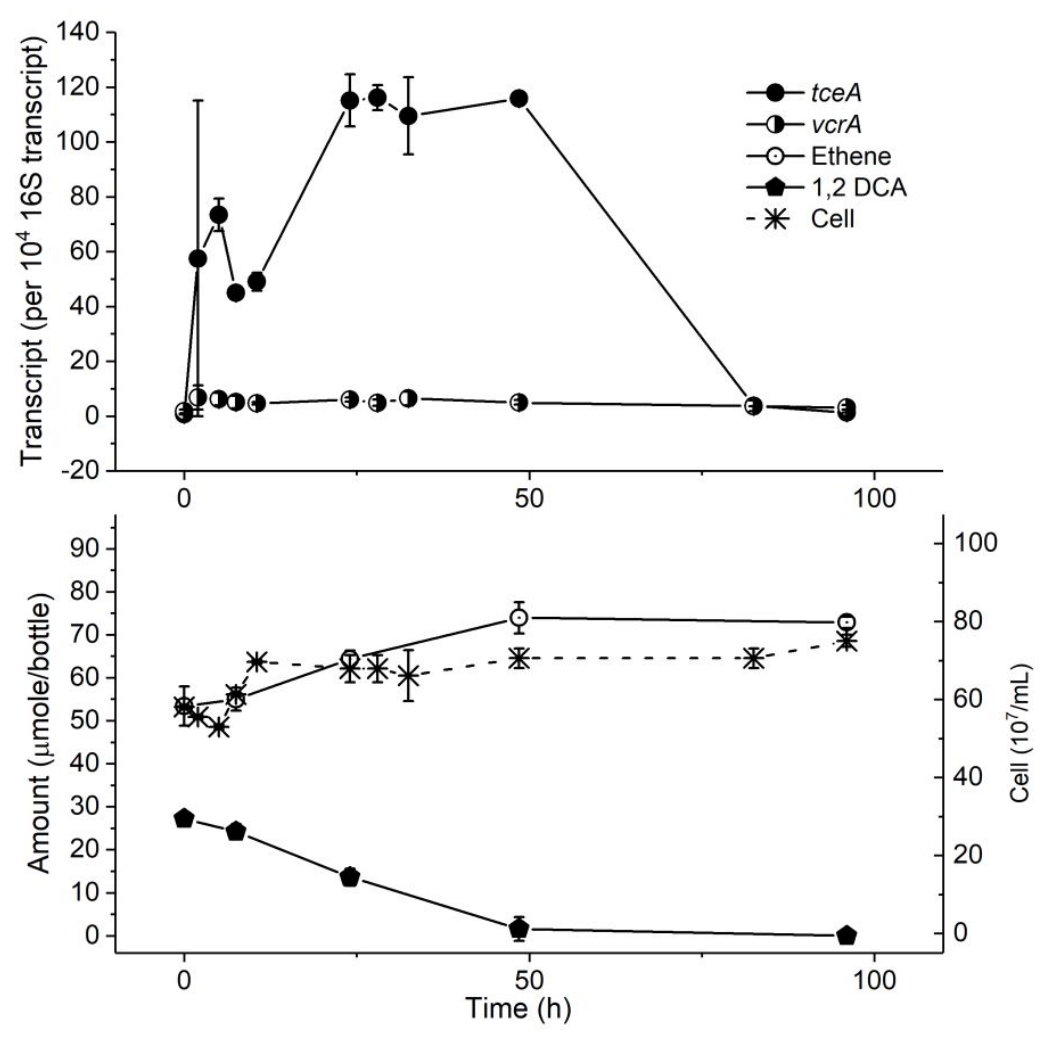




\section{Supporting Tables}

Supplementary Table S1. Primers used in this study

\begin{tabular}{|c|c|c|c|}
\hline Primer ID & Sequence (5'-3') & Target & Reference \\
\hline $341 \mathrm{~F}-\mathrm{GC}$ & $\begin{array}{l}\text { CGCCCGCCGCGCGCGGCGGGCG } \\
\text { GGGCGGGGGCACGGGGGGCCTA } \\
\text { CGGGAGGCAGCAG }\end{array}$ & \multirow[t]{2}{*}{ Bacterial 16S rRNA, DGGE } & (1) \\
\hline $518 \mathrm{R}$ & ATTACCGCGGCTGCTGG & & \\
\hline Dhc_qF2 & GGTAATACGTAGGAAGCAAGCG & \multicolumn{2}{|l|}{$\begin{array}{l}\text { Dehalococcoides 16S rRNA, (2) } \\
\text { qPCR }\end{array}$} \\
\hline Dhc_qR & CCGGTTAAGCCGGGAAATT & & \\
\hline GEO12-1F & TCAACCCAGGGCTCAAATG & GEO12 rdhl, qPCR & this study \\
\hline GEO12-1R & CCGATAAGCTGATAACCCAAAT & & \\
\hline GEO12-2F & GCTGTGAGCAGGTGGGTATCT & GEO12 rdh2, qPCR & this study \\
\hline GEO12-2R & AATTTGCATGTCCGGTGGTA & & \\
\hline GEO12-3F & TGCTTCGCTTGTTCGTTCTG & GEO12 rdh3, qPCR & this study \\
\hline GEO12-3R & CCCCATTTCGGACACCCTA & & \\
\hline GEO12-4F & GTTTCCCACCGCATACAGG & GEO12 rdh4, qPCR & this study \\
\hline GEO12-4R & TTGGAGAAAGAGGCAAGTCAGT & & \\
\hline GEO12-5F & GTTCGCACCCTCTTTTACAATG & GEO12 rdh5, qPCR & this study \\
\hline GEO12-5R & ATGGAAGTGGTTGAAACGGTAG & & \\
\hline tceA_1270F & ATCCAGATTATGACCCTGGTGAA & tce $A, \mathrm{qPCR}$ & (3) \\
\hline tceA_1336R & GCGGCATATATTAGGGCATCTT & & \\
\hline vcrA11F & GTATGGTCCGCCACATGATTC & vcr $A, \mathrm{qPCR}$ & (4) \\
\hline vcrA11R & TCTTCTGGAGTACCCTCCCATTT & & \\
\hline GEO12-rpoBF & CATTTATCACCAGCGGTACG & GEO12 rрoв, qPCR & this study \\
\hline GEO12-rpoBR & GCCGGGTCATCTGAAATAGT & & \\
\hline luc-F & TACAACACCCCAACATCTTCGA & luciferase, qPCR & (5) \\
\hline luc-R & GGAAGTTCACCGGCGTCAT & & \\
\hline GEO-vcrA F & AGCATAGTTAGTCCAACTGCTAA & full-length $v c r A$ & this study \\
\hline GEO-vcrA R & TGGCTCTAGGGAAAGACCTAA & & \\
\hline GEO-tceA F & CCGCCAATAAGCATACCGAC & full-length $t c e A$ & this study \\
\hline GEO-tceA R & GCTTGCTGACTACCGCATGA & & \\
\hline
\end{tabular}


Supplementary Table S2. Bootstrap hypothesis testing of gene expression

\begin{tabular}{|c|c|c|c|c|c|c|c|c|c|c|c|}
\hline \multirow[b]{2}{*}{ Gene } & \multicolumn{3}{|c|}{$\begin{array}{c}\text { Expression level }\left(e_{1}\right) \text { at } \\
\text { condition } 1\end{array}$} & \multicolumn{3}{|c|}{$\begin{array}{l}\text { Expression level }\left(e_{2}\right) \text { at } \\
\text { condition } 2\end{array}$} & \multicolumn{5}{|c|}{$\begin{array}{c}\text { Percentile of } \Delta=e_{1}-e_{2}, \text { bootstrap } \\
\text { repeats }=2000\end{array}$} \\
\hline & Condition 1 & $\begin{array}{l}\text { Mea } \\
\text { per }\end{array}$ & & Condition 2 & $\begin{array}{l}\text { Mean } \\
\text { per 10 }\end{array}$ & & 0.5 & 2.5 & 50 & 97.5 & 99.5 \\
\hline \multirow[t]{4}{*}{ tceA } & $\begin{array}{l}\text { GEO12 } \\
\text { no CF }\end{array}$ & 22.1 & 14.9 & $\begin{array}{l}\text { GEO12 } \\
\text { w/ CF }\end{array}$ & 12.2 & 6.9 & -21.0 & -16.8 & 7.7 & 42.4 & 51.3 \\
\hline & $\begin{array}{l}\text { GEO12 } \\
\text { no CF }\end{array}$ & 22.1 & 14.9 & $\begin{array}{l}\text { GEO12CF } \\
\text { no } \mathrm{CF}\end{array}$ & 6.5 & 4.2 & -10.8 & -8.2 & 13.4 & 47.6 & $\overline{51.6}$ \\
\hline & $\begin{array}{l}\text { GEO12 } \\
\text { w/ CF }\end{array}$ & 12.2 & 6.9 & $\begin{array}{l}\text { GEO12CF } \\
\text { w/ CF }\end{array}$ & 65.2 & 34.1 & -128 & -120 & -49.4 & -3.3 & 5.1 \\
\hline & $\begin{array}{l}\text { GEO12CF } \\
\text { no } \mathrm{CF}\end{array}$ & 6.5 & 4.2 & $\begin{array}{l}\text { GEO12CF } \\
\text { w/ CF }\end{array}$ & 65.2 & 34.1 & -128 & $\underline{-125}$ & -55.4 & -12.9 & -4.4 \\
\hline \multirow[t]{4}{*}{ verA } & $\begin{array}{l}\text { GEO12 } \\
\text { no CF }\end{array}$ & 49.1 & 19.1 & $\begin{array}{l}\text { GEO12 } \\
\text { w/ CF }\end{array}$ & 27.7 & 13.5 & -30.3 & -20.5 & 21.2 & 74.2 & 81.2 \\
\hline & $\begin{array}{l}\text { GEO12 } \\
\text { no CF }\end{array}$ & 49.1 & 19.1 & $\begin{array}{l}\text { GEO12CF } \\
\text { no CF }\end{array}$ & 36.2 & 12.2 & -34.6 & -25.2 & 10.8 & 63.0 & 78.5 \\
\hline & $\begin{array}{l}\text { GEO12 } \\
\mathrm{w} / \mathrm{CF}\end{array}$ & 27.7 & 13.5 & $\begin{array}{l}\text { GEO12CF } \\
\text { w/ CF }\end{array}$ & 143.7 & 75.5 & -266 & -258 & -117 & 6.2 & 19.4 \\
\hline & $\begin{array}{l}\text { GEO12CF } \\
\text { no } \mathrm{CF}\end{array}$ & 36.2 & 12.2 & $\begin{array}{l}\text { GEO12CF } \\
\text { w/ CF }\end{array}$ & 143.7 & 75.5 & -261 & -246 & -109 & 13.6 & 17.9 \\
\hline
\end{tabular}

Note: Statistical analysis is based on the same dataset as Figure 5, excluding data points of time zero (when induction of transcripts had not yet started) and time points when TCE was depleted.

Differences of expression levels were not significant if the percentile range $(2.5,97.5)$ spanned the 0 point. Significant differences were underlined. 
Supplementary Table S3. Average nucleotide identity calculated using MUMMer (ANIm) and DNADNA hybridization (DDH) values of Dehalococcoides genomes compared to the genome of Dehalococcoides mccartyi strain GEO12.

\begin{tabular}{llll}
\hline Subgroup & GEO12 vs. & DDH [\%] & ANIm [\%] \\
\hline Cornell & CG4 & 83.9 & 98.3 \\
& 195 & 82.3 & 98.1 \\
\hline Victoria & UCH007 & 39.7 & 91.0 \\
& VS & 39.2 & 90.8 \\
& GY50 & 39.1 & 90.8 \\
& CG1 & 38.8 & 90.7 \\
\hline Pinellas & BTF08 & 32.3 & 87.8 \\
& GT & 31.1 & 87.1 \\
& 11a5 & 30.5 & 86.9 \\
& Ibaraki & 30.5 & 86.9 \\
& CG5 & 30.4 & 86.9 \\
& DCMB5 & 30.2 & 86.9 \\
& BAV1 & 30.2 & 86.8 \\
& CBDB1 & 30.0 & 86.7 \\
\hline
\end{tabular}




\section{Supplementary Data File S1}

Transcripts of seven $r d h A$ genes, 16S rRNA, and $r p o B$ during dechlorination of TCE, 1,2-DCA, and trans-DCE by Dehalococcoides mccartyi strain GEO12 without chloroform, dechlorination of TCE with chloroform by Dehalococcoides mccartyi strain GEO12, and dechlorination of TCE with and without chloroform by culture GEO12CF.

\section{References}

(1) Muyzer, G.; Dewaal, E. C.; Uitterlinden, A. G., Profiling of complex microbial populations by denaturing gradient gel electrophoresis analysis of polymerase chain reaction-amplified genes coding for 16S rRNA. Applied and Environmental Microbiology 1993, 59 (3), 695-700.

(2) Holmes, V. F.; He, J.; Lee, P. K. H.; Alvarez-Cohen, L., Discrimination of multiple Dehalococcoides strains in a trichloroethene enrichment by quantification of their reductive dehalogenase genes. Applied and Environmental Microbiology 2006, 72 (9), 5877-5883.

(3) Johnson, D. R.; Lee, P. K. H.; Holmes, V. F.; Fortin, A. C.; Alvarez-Cohen, L., Transcriptional expression of the tceA gene in a Dehalococcoides-containing microbial enrichment. Applied and Environmental Microbiology 2005, 71 (11), 7145-7151.

(4) Lee, P. K. H.; Cheng, D.; West, K. A.; Alvarez-Cohen, L.; He, J., Isolation of two new Dehalococcoides mccartyi strains with dissimilar dechlorination functions and their characterization by comparative genomics via microarray analysis. Environmental Microbiology 2013, 15 (8), 2293-2305.

(5) Johnson, D. R.; Lee, P. K. H.; Holmes, V. F.; Alvarez-Cohen, L., An internal reference technique for accurately quantifying specific mRNAs by real-time PCR with application to the tceA reductive dehalogenase gene. Applied and Environmental Microbiology 2005, 71 (7), 3866-3871. 